\title{
Determination on the Structure of Au Nanorods with Pentagonal Cross-Sections by Various TEM Techniques
}

\author{
Lihua Zhang ${ }^{1}$, Matthew A. Wall ${ }^{2,3}$, Stefan Harmsen ${ }^{2}$, Charles Michael Drain ${ }^{3}$, Moritz F. Kircher ${ }^{2,4,5}$ \\ 1. Center for Functional Nanomaterials, Brookhaven National Laboratory, Upton, NY, 11973, USA. \\ 2. Department of Radiology, Memorial Sloan-Kettering Cancer Center, New York, NY, 10065, USA. \\ 3. Department of Chemistry, Hunter College of the City University of New York, New York, NY. \\ 10065, USA.
}

4. Nanotechnology Center, Memorial Sloan-Kettering Cancer Center, New York, NY, 10065, USA.

5. Department of Radiology, Weill, Cornell Medical College, New York, NY 10065, USA.

Noble metal nanostructures have drawn increasing interest due to potential applications in catalysis, biology, electronics, etc. It has been demonstrated that the optical, biological, and chemical properties are highly influenced by morphology and size $[1,2]$. In this work, we present an investigation on the structure of multi-twinned Au nanorods using various electron microscopy techniques.

Fig.1(a) and (c) are bright field transmission electron microscopy(TEM) images of typical Au nanorods. Fig.(b) and (d) are corresponding electron patterns of the structures presented in of Fig.(a) and (c). Electron diffraction in Fig.1(b) indicates a superposition of the [110] and [111] zone of a face-centered cubic(fcc) structure, while electron diffraction from Fig.1(d) shows another superposition of [100] and [112] zone of fcc structure. These electron diffraction patterns could not be indexed as single pattern, which indicates the nanorod is not a single domain crystal.

High resolution TEM (HRTEM) images along the orientations shown in Fig.1(b) and Fig.1(d) were taken to get more information regarding the structure of these Au nanorods. A domain boundary is seen on HRTEM images of those nanorods with diffraction patterns such as shown Fig.1(b). One part of the rod is along [110] orientation and the other part is along the [111] orientation, as confirmed by Fast Fourier Transforms (FFT) of the images. HRTEM images of those nanorods with diffraction patterns such as shown Fig.1(d) show a boundary too, while the FFT of each part doesn't show a single crystal pattern. Dark field TEM images were observed to further understand the structure of Au nanorod. On diffraction of [110] and [111] type, dark field images show two separate domains on each side. However, on the [100] and [112] type diffraction, the overlapping between two excited orientations were found in dark field images. This is consistent with the result of HRTEM images.

Electron diffraction analysis and HRTEM images of nanorods showed superpositions of two specific pairs of crystallographic zones, either [110] and [111] or [100] and [112], which is in good agreement with a fcc penta-twinned crystal with five $\{111\}$ twin boundaries, arranged radially to the [110] direction of elongation. The cross-sectional models are also shown in Fig.2, with respect to the electron beam. Dark field analysis of both electron diffraction types also confirmed the multi-twinned structure.

[1] J R Morones, Nanotechnology, 16(2005), p. 2346.

[2] R J Chimentao et al, Chem. Commun. 7 (2004), p. 846.

[3] J.Reyes-Gasga et al, J Crystal Growth, 286 (206), p.162.

[4] J L Elechiguerra et al, J Mater. Chem. 16 (2006), p. 3906. 
[5] TEM Research carried out at the Center for Functional Nanomaterials, Brookhaven National Laboratory, which is supported by the U.S. Department of Energy, Office of Basic Energy Sciences, under Contract No. DE-AC02-98CH10886.

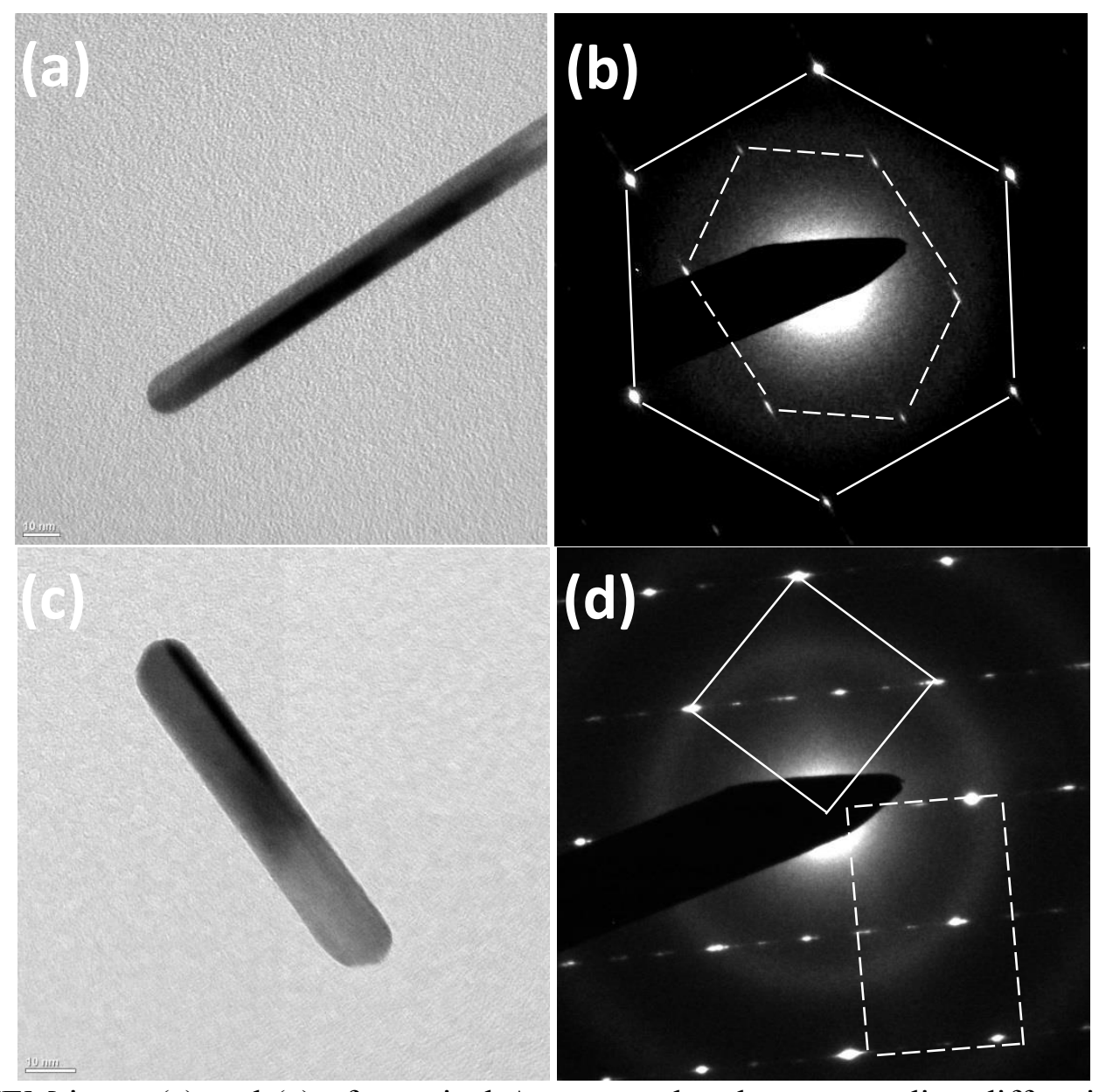

Figure 1. TEM image(a) and (c) of a typical Au nanorod and corresponding diffraction (b) with [110] and [111] superposition, and (d) with [100] and [112] superposition.
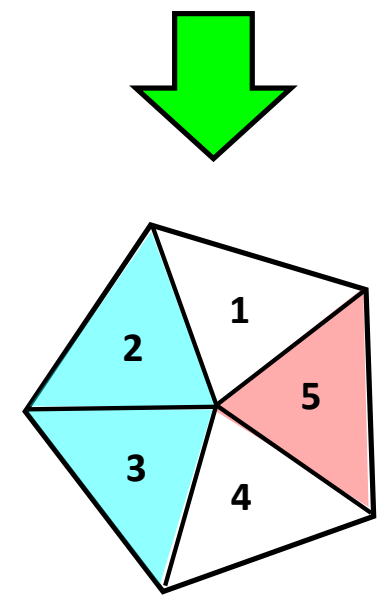

\section{Electron beam}

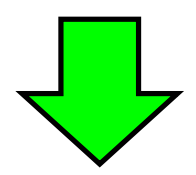

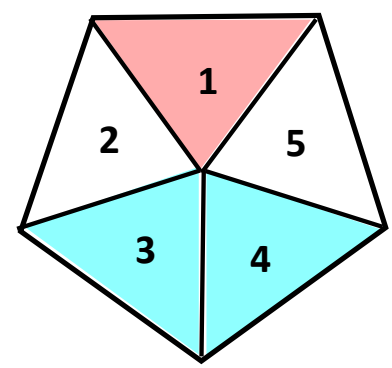

Figure 2. Cross-sectional models of Au nanorod show twin structure with respect to electron beam. 